\title{
Fetal Isolated Anomalous Origin of Right Pulmonary Artery from Aorta
}

\author{
Shi Zeng, PhD ${ }^{1}$ Qichang Zhou, $\mathrm{PhD}^{1} \quad$ jiawei Zhou, MD ${ }^{1} \quad$ Qinghai Peng, $\mathrm{PhD}^{1}$
}

1 Department of Ultrasonography, The Second Xiangya Hospital, Central South University, Changsha, Hunan, People's Republic of China

Am J Perinatol Rep 2015;5:e80-e81.
Address for correspondence Qichang Zhou, PhD, Department of Ultrasound, Second Xiangya Hospital of Central South University, 139 Renmin Road (M), Changsha 410011, China (e-mail: hnzqc2013@163.com).

\begin{abstract} Keywords

- anomalous origin of pulmonary artery from aorta

- fetal echocardiography

- prenatal diagnosis

The anomalous origin of a branch pulmonary artery from the aorta (AOPA) is characterized by the anomalous origin of one of the branch pulmonary arteries (PA) from the ascending aorta and a normal origin of the other PA from main PA. AOPA is an extremely rare cardiac malformation. Few studies have reported fetal anomalous origin of PA from aorta with other malformation. We report a case of isolated distal anomalous origin of the right PA from the aorta that was diagnosed by fetal echocardiography at 25 weeks' of gestation. Tracing the course of PA branches is important to make diagnosis.
\end{abstract}

\section{Case}

A 27-year-old, gravida 1 woman was referred to our center for fetal echocardiography at 25 weeks and 4 days following routine ultrasound screening with suspected cardiac anomaly. The family history was negative for congenital heart malformations. The heart had a normal cardiac axis with situs solitus, concordant atrioventricular, and ventriculoarterial connections. The main pulmonary artery $(\mathrm{PA})$ was continuous with the left PA and there was no confluence with the right PA. On detailed examination, it was noted that the right PA arose anomalously from the ascending aorta originating near the base of the brachiocephalic artery (-Figs. 1-3). Cordocentesis had yielded a normal karyotype (46, XY) and the FISH analysis for deletion 22q11.2 had yielded normal results. The pregnancy was uneventful, and at 39 weeks' gestation, a male infant was delivered vaginally.

Neonatal echocardiographic examination confirmed the aforementioned findings. Surgical repair was performed at 1 month through a median sternotomy. Briefly, under cardiopulmonary bypass (CPB), an end-to-side anastomosis was performed between the aberrant PA and the main PA directly. The right PA and aorta remains patent with no evidence of stenosis by echocardiography during regular visits to the outpatient clinic.

received

December 30, 2014 accepted after revision January 23, 2015 published online March 4, 2015

\section{Discussion}

The anomalous origin of a branch PA from the aorta (AOPA), is characterized by the anomalous origin of one of the branch pulmonary arteries from the ascending aorta and a normal origin of the other PA from the right ventricular outflow tract (RVOT) in the presence of two semilunar valves. ${ }^{1}$ AOPA is an extremely rare cardiac malformation which accounts for $0.12 \%$ of all congenital heart defects. ${ }^{2}$ Although anomalous origin of the right pulmonary branch (AORPA) is five to six times more frequent than that of the left (AOLPA), ${ }^{1}$ the latter is the more commonly associated lesion with either TOF or right aortic arch. $^{3}$ In most cases, the anomalous branch originates on the posterolateral wall of the ascending aorta. In $15 \%$ of cases, the origin is distal near the base of the innominate artery. ${ }^{4}$

In babies with AOPA, the affected lung receives unrestricted blood at systemic pressure from the aorta, whereas the contralateral lung receives the entire right ventricular cardiac output. If left untreated, 1 -year survival may be as low as $30 \% 5$ because of pulmonary vascular obstructive disease and heart failure. Surgical management early in life results in an excellent hemodynamic and anatomic results and improved the outcomes in these patients. ${ }^{6}$ Therefore, early diagnosis even in fetal period is essential to perform early postnatal surgical repair and counsel the family at the time of diagnosis. Tel: +1(212) 584-4662.
Copyright $\odot 2015$ by Thieme Medical Publishers, Inc., 333 Seventh Avenue, New York, NY 10001, USA.

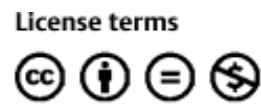

$10.1055 / \mathrm{s}-0035-1547331$ ISSN 2157-6998. 


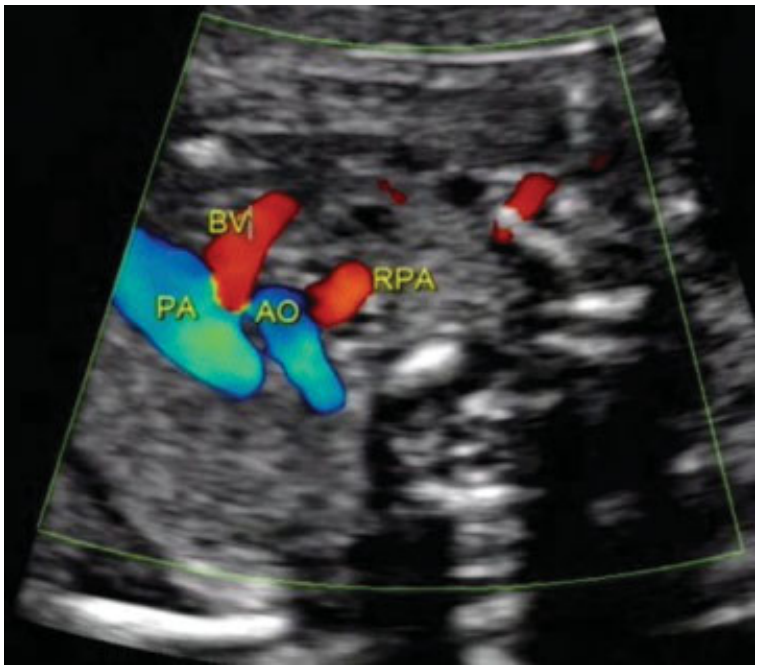

Fig. 1 The three vessel view showed right pulmonary artery originated from the ascending aorta. AO, aorta; $\mathrm{BV}$, brachiocephalic vein; $\mathrm{PA}$, main pulmonary artery; RPA, right pulmonary artery.

To our knowledge, there were only three cases diagnosed by fetal echocardiography. ${ }^{3,4,7}$ When the normal "Y"-shaped pulmonary branch view is not displayed in fetal cardiac screening, tracing the course of each PA branch is very essential to make appropriate diagnosis. The key characteristics of AOPA in fetal echocardiography are as follows: (1) the main PA is continuous with one PA and there is no confluence with the other PA; (2) the anomalous PA arising from the ascending aorta is usually present in the three vessel/three vessel-trachea view, and aortic arch oblique coronal view. Sometimes, reverse tracing PA from the hilus of the affected lung is also helpful to demonstrate the course and origin of PA.

It is important to distinguish this anomaly from other conotruncal malformation, such as, pulmonary atresia, transposition of great arteries, and truncus arteriosus. In cases with pulmonary atresia, the main PA and branches are present

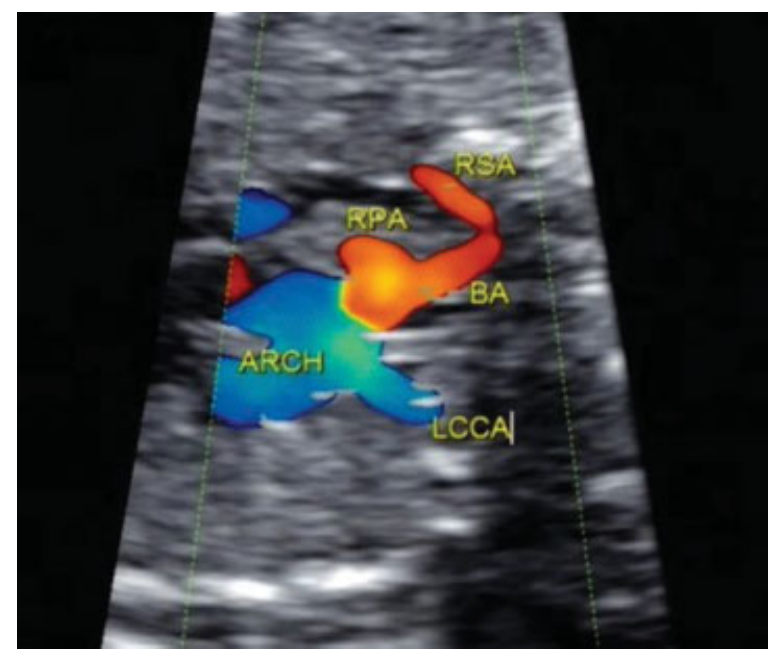

Fig. 2 The aortic arch oblique coronal view showed the origin of right pulmonary artery was near the base of the brachiocephalic artery. $\mathrm{ARCH}$, aortic arch; BA, brachiocephalic artery; LCCA, left common carotid artery; RPA, right pulmonary artery; RSA, right subclavic artery.

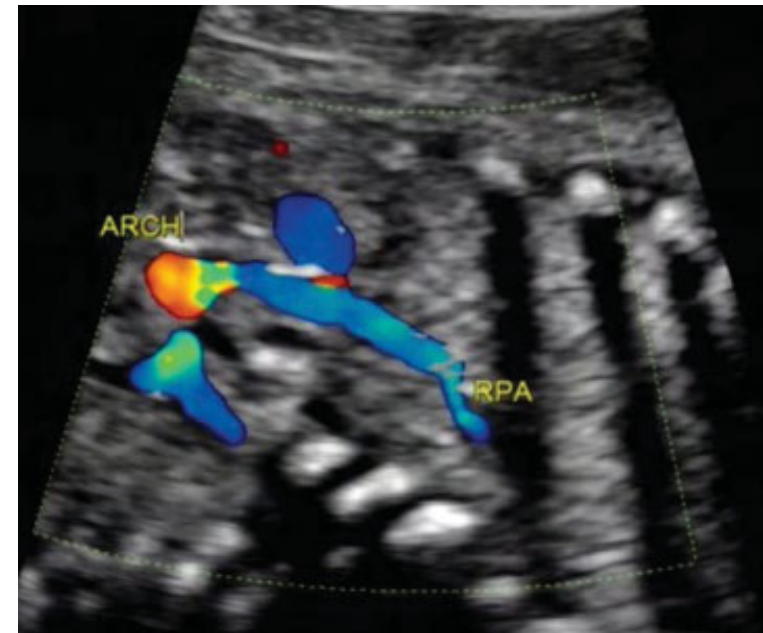

Fig. 3 The overall course of the right pulmonary artery, originating from the lateral wall of ascending aorta and entering lung tissue, was demonstrated. ARCH, aortic arch; RPA, right pulmonary artery.

whereas blood supply to one or both pulmonary arteries originates from major aortopulmonary collaterals or from the ductus arteriosus. When the anomalous origin of PA arises near the aortic valve, the aorta could be mistaken as "main PA with branches" and can be diagnosed as transposition of the great arteries. As for truncus arteriosus, there is only one semilunar valve at the base of the heart.

\section{Acknowledgment}

This study was supported by the National Clinical Key Subject (Medical Imageology) Construction Project of China (2013(544)).

\section{References}

1 Garg P, Talwar S, Kothari SS, et al. The anomalous origin of the branch pulmonary artery from the ascending aorta. Interact Cardiovasc Thorac Surg 2012;15(1):86-92

2 Kutsche LM, Van Mierop LH. Anomalous origin of a pulmonary artery from the ascending aorta: associated anomalies and pathogenesis. Am J Cardiol 1988;61(10):850-856

3 Diab K, Richardson R, Pophal S, Alboliras E. Left hemitruncus associated with tetralogy of fallot: fetal diagnosis and postnatal echocardiographic and cardiac computed tomographic confirmation. Pediatr Cardiol 2010;31(4):534-537

4 Oztunç F, Güzeltaş A. Prenatal diagnosis of anomalous origin of the right pulmonary artery from the ascending aorta with hypoplastic right ventricle and pulmonary stenosis. Ultrasound Obstet Gynecol 2008;32(4):592-593

5 Fontana GP, Spach MS, Effmann EL, Sabiston DC Jr. Origin of the right pulmonary artery from the ascending aorta. Ann Surg 1987; 206(1):102-113

6 Nathan M, Rimmer D, Piercey G, et al. Early repair of hemitruncus: excellent early and late outcomes. J Thorac Cardiovasc Surg 2007; 133(5):1329-1335

7 Jung MJ, Yoo SJ. Prenatal diagnosis of anomalous origin of the right pulmonary artery from the ascending aorta. Cardiol Young 2002; 12(2):186-188 